\title{
Design Optimization of Pixel Sensors using Device Simulations for the Phase-II CMS Tracker Upgrade
}

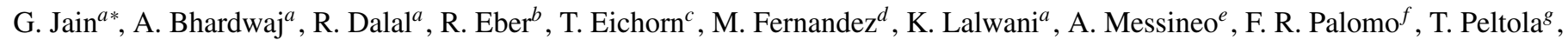 \\ M. Printz ${ }^{b}$, K. Ranjan ${ }^{a}$, I. Villa $^{d}$, S. Hidalgo ${ }^{h}$
}

CDRST, Department of Physics $\mathcal{E}$ Astrophysics, University of Delhi, Delhi, India ${ }^{a}$; Institute fur Experimentelle Kernphysik, Germany ${ }^{b}$; Deutsches Elektronen Synchrotron, Germany ${ }^{c}$; Instituto de Fisica de Cantabria, Spain ${ }^{\text {; }}$ Universita di Pisa E INFN sez. di Pisa, Italy ${ }^{e}$; Escuela Superior de Ingenieros, Universidad de Sevilla, Spain $^{f}$; Helsinki Institute of Physics, Finland ${ }^{g}$; Instituto de Microelectronica de Barcelona, Centro Nacional de Microelectronica, Spain ${ }^{h}$

On behalf of the CMS Collaboration

\begin{abstract}
In order to address the problems caused by the harsh radiation environment during the high luminosity phase of the LHC (HL-LHC), all silicon tracking detectors (pixels and strips) in the CMS experiment will undergo an upgrade. And so to develop radiation hard pixel sensors, simulations have been performed using the 2D TCAD device simulator, SILVACO, to obtain design parameters. The effect of various design parameters like pixel size, pixel depth, implant width, metal overhang, p-stop concentration, p-stop depth and bulk doping density on the leakage current and critical electric field are studied for both non-irradiated as well as irradiated pixel sensors. These 2D simulation results of planar pixels are useful for providing insight into the behaviour of non-irradiated and irradiated silicon pixel sensors and further work on 3D simulation is underway.
\end{abstract}

Keywords: CMS, tracker detector, silicon sensor, high energy physics

PACS: 29.40.Cs, 29.40.Gx

\section{Introduction}

The precise track reconstruction and accurate vertex identification in High Energy Physics experiments requires silicon pixel detectors. In the High Luminosity upgrade of the LHC (HL-LHC) envisaged for the year 2023, the instantaneous luminosity is expected to increase from $300 \mathrm{fb}^{-1}$ to $3000 \mathrm{fb}^{-1}$, which would demand radiation harder detectors for the CMS tracker [1]. Hence, a new radiation hard design has to be devised through careful optimization of different geometry parameters. In this work, a stepwise approach for identification of such a design, through the TCAD device simulator Silvaco [2], using 2D structures, is described.

\section{Pixel Geometry and Simulation Structure}

Simulations were carried out on two n-on-p planar pixel sensor geometries, $50 \times 50 \mu \mathrm{m}^{2}$ and $100 \times 25 \mu \mathrm{m}^{2}$, for two wafer thicknesses of $150 \mu \mathrm{m}$ and $200 \mu \mathrm{m}$. Each geometry has two configurations, which are referred to as 'normal' and 'wide' having different implant widths as shown in Figure 1 on p-stop and p-spray isolation techniques. In simulations, a p-type silicon substrate with a uniform doping concentration of $3 \times 10^{12}$ $\mathrm{cm}^{-3}$ and $\mathrm{n}^{+}$implants with a Gaussian profile with a peak concentration of $1 \times 10^{19} \mathrm{~cm}^{-3}$ and a junction depth equal to 1.5 $\mu \mathrm{m}$ are considered. A schematic of the simulated pstop isolation normal structure is shown in Figure 2. The simulation

\footnotetext{
${ }^{*}$ Corresponding author

Email address: geetikajain. hep@gmail.com (G. Jain $\left.{ }^{a}\right)$
}

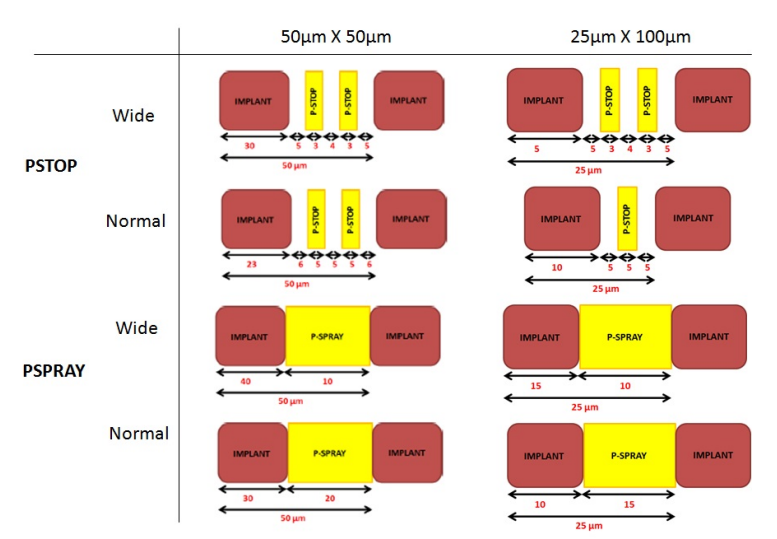

Figure 1: Schematics of the pixel geometries with different configurations.

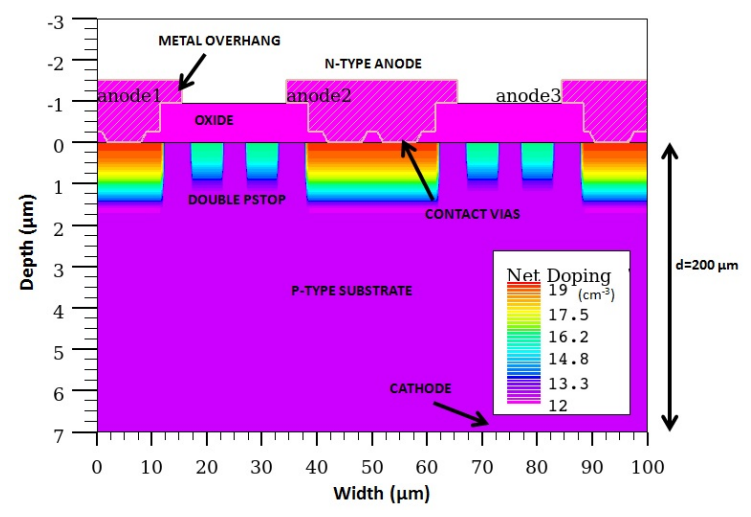

Figure 2: Device simulation structure for p-stop isolation studies. 
Table 1: Bulk and surface radiation damage trap model.

\begin{tabular}{cccccc}
\hline \hline Damage & Trap type & $\begin{array}{c}\text { Energy level } \\
(\mathrm{eV})\end{array}$ & $\begin{array}{c}\text { Density } \\
\left(\mathrm{cm}^{-3}\right)\end{array}$ & $\begin{array}{c}\sigma_{e} \\
\left(\mathrm{~cm}^{-2}\right)\end{array}$ & $\begin{array}{c}\sigma_{h} \\
\left(\mathrm{~cm}^{-2}\right)\end{array}$ \\
\hline Bulk & Acceptor & $E_{C}-0.51$ & $4 \times \phi$ & $2.0 \times 10^{-14}$ & $2.6 \times 10^{-14}$ \\
Bulk & Donor & $E_{V}+0.48$ & $3 \times \phi$ & $2.0 \times 10^{-14}$ & $2.0 \times 10^{-14}$ \\
Surface & Acceptor & $E_{C}-0.60$ & $0.6 \times Q_{F}$ & $0.1 \times 10^{-14}$ & $0.1 \times 10^{-14}$ \\
Surface & Acceptor & $E_{C}-0.39$ & $0.4 \times Q_{F}$ & $0.1 \times 10^{-14}$ & $0.1 \times 10^{-14}$
\end{tabular}
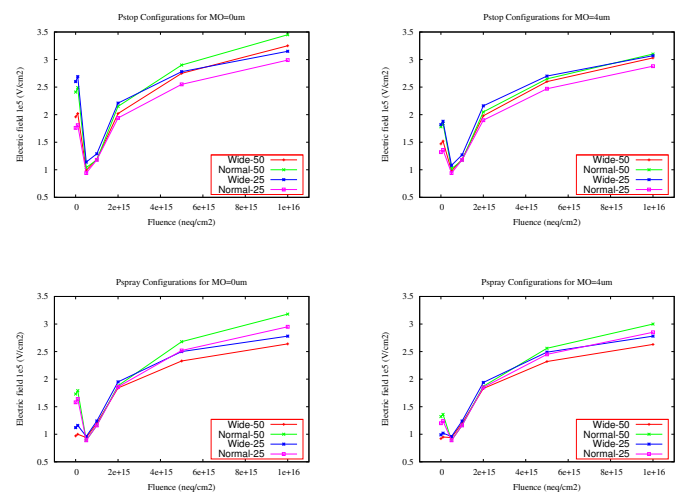

Figure 3: The maximum electric field is plotted as a function of fluence for all the 8 designs with sensor thickness of $200 \mu \mathrm{m}$ at a bias voltage of $1000 \mathrm{~V}$.

of non-irradiated pixel sensors is performed by incorporating a fixed oxide charge density $\left(Q_{F}\right)$ of $1 \times 10^{11} \mathrm{~cm}^{-2}$ and two interface traps at the $\mathrm{Si} / \mathrm{SiO}_{2}$, with interface density $\mathrm{N}_{i t}$. Irradiated pixel sensors are simulated by implementing two bulk trap levels along with appropriate values of $Q_{F}$ and $N_{i t}$ for different fluences considering the level of radiation damage to pixel sensors in HL-LHC scenario [3]. Table 1 lists the energy levels, density of traps as a product of a constant term (called introduction rate) times the $Q_{F}$ / fluence $(\phi)$ and carrier capture cross sections $\left(\sigma_{e}\right.$, $\sigma_{h}$ ) used for the bulk and surface damage, respectively, in the simulations.

\section{Simulation Results}

The electric field has been studied as a function of fluence for different design options, to compare the radiation hardness. The critical electric field is analyzed for different configurations and it is found that the maximum electric field is at $0.9 \mu \mathrm{m}$ below the $\mathrm{Si} / \mathrm{SiO}_{2}$ interface, near the implant curvature. Knowing the critical electric field is important because the geometry parameters are optimized looking at the operation stability against breakdown caused by the critical electric field in the sensor. In Figure 3, the maximum electric field is plotted as a function of fluence for all the 8 designs both with and without metal overhang (MO). It is found that of all the designs, p-stop normal configuration of pitch $25 \mu \mathrm{m}$ and p-spray wide configuration of pitch $50 \mu \mathrm{m}$, have the least increase in electric field for increasing fluence. For these configurations, the effect of sensor thickness $(d)$ and p-stop/p-spray implant depth $\left(D_{p s t-p s p}\right)$, for a constant $\mathrm{p}$-stop/p-spray concentration $\left(N_{p s t-p s p}\right)$ of $1 \times 10^{16} /$ $1 \times 10^{15} \mathrm{~cm}^{-3}$, on the maximum electric field for different fluences for structures with and without MO is plotted in Figure
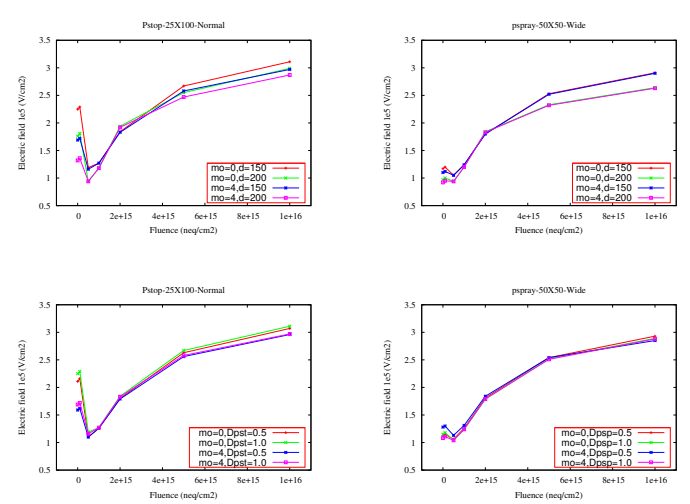

Figure 4: The effect of the variation of different geometry parameters is shown for the best p-stop \& p-spray designs as concluded from Figure 3. The sensor thickness is varied for constant $\mathrm{p}$-stop/p-spray depth of $1 \mu \mathrm{m}$ in top plots, whereas the p-stop/p-spray depth is varied for a fixed sensor thickness of 150 $\mu \mathrm{m}$ in bottom plots, with and without MO.

4. It is seen that for p-stop normal configuration of pitch $25 \mu \mathrm{m}$ the electric field reduces for larger sensor thickness and for the design with a MO of $4 \mu \mathrm{m}$. Also, the plot clearly shows that for the same configuration the effect of $D_{p s t}$ on the maximum electric field is negligible. On the other hand for the p-spray wide configuration of pitch $50 \mu \mathrm{m}$ the electric field reduces for larger sensor thickness, but there is no effect of MO. Further, it may be noted that for this configuration the maximum electric field is insensitive to the variation in $D_{p s p}$.

\section{Summary}

2-D simulations have been performed to optimize the pixel sensor design for different configurations. Out of the 8 simulated designs, p-stop normal configuration with pitch $25 \mu \mathrm{m}$ and p-spray wide configuration with pitch $50 \mu \mathrm{m}$ are the most radiation tolerant. The optimized set of parameters is : $d=$ $200 \mu \mathrm{m}$ with a p-stop/p-spray depth of $1 \mu \mathrm{m}$ and a MO of 4 $\mu \mathrm{m}$. However, a further study on charge collection efficiency is needed to finalize the layout parameters and further work on $3 \mathrm{D}$ simulation is underway.

\section{Acknowledgments}

The authors are thankful to the support of CDRST, DU and DST, UGC-JRF for providing research grant.

\section{References}

[1] CMS Collaboration, The CMS experiment at the CERN LHC, 2008 JINST 3 S08004.

[2] ATLAS Silvaco version 5.15.32.R Nov 2009, Users manual. http://www.silvaco.com

[3] R. Dalal et al., Simulation of Irradiated Si Detectors, PoS(Vertex2014) 030 . 